\title{
Left Atrial Myxoma Masquerading as Pulmonary Embolism on Electrocardiogram: A Case Report
}

\author{
Anton Mararenko ${ }^{\mathrm{a}, \mathrm{c}}$, Ndausung Udongwo ${ }^{\mathrm{b}}$, Dhaval Desai ${ }^{\mathrm{b}}$, Matthew S. Schoenfeld ${ }^{\mathrm{b}}$
}

\begin{abstract}
Primary cardiac tumors are an incredibly rare finding. Cardiac myxomas are the most primary cardiac tumors that often occur within the left atrium. When left untreated, they pose a high risk of causing hemodynamic collapse by obstruction or can embolize and result in thromboembolic stroke. The presentation of cardiac myxoma varies greatly and can be associated with significant morbidity and mortality when undiagnosed. A careful physical examination and high degree of suspicion is crucial in early diagnosis and intervention. Our team presents a 46-year-old female patient with no significant past medical history that presented to the emergency department with a neurological deficit that was concerning for a transient ischemic attack. Initial laboratory workup and electrocardiogram was suggestive for pulmonary embolism; however, upon evaluation with imaging, the patient was found to have a $1.6 \times 3.4 \mathrm{~cm}$ mass fixed to the mitral leaflet that was then confirmed on transthoracic echocardiography. Our patient was found to have non-obstructive coronary artery disease on cardiac catheterization and ultimately underwent successful mass resection by cardiothoracic surgery.
\end{abstract}

Keywords: Atrial myxoma; Pulmonary embolism; Benign cardiac tumor; Thromboembolic infarct

\section{Introduction}

Primary cardiac tumors (PCTs) are less common than metastatic cancers to the heart [1]. The latter is commonly associated with carcinoma of the lungs [2]. PCTs are rare with a reported incidence of $0.00138-0.03 \%[1,2]$. More than $75 \%$ of PCTs are benign with myxoma being the most common, particularly

Manuscript submitted August 28, 2021, accepted October 5, 2021

Published online December 2, 2021

${ }^{a}$ Department of Internal Medicine, Jersey Shore University Medical Center, Neptune, NJ, USA

bepartment of Cardiology, Jersey Shore University Medical Center, Neptune, NJ, USA

'Corresponding Author: Anton Mararenko, Department of Internal Medicine, Jersey Shore University Medical Center, Neptune, NJ, USA.

Email: Anton.Mararenko@hmhn.org

doi: https://doi.org/10.14740/jmc3775 those occurring in the left atrium $[1,2]$. Atrial myxomas have been reported to cause a constellation of embolic complications including bilateral pulmonary embolism (PE), cerebrovascular accidents, myocardial infarctions, splenic or renal infarctions [2]. The clinical presentation is variable, ranging from an incidental finding in asymptomatic patients to those presenting with shock or sudden cardiac death [2]. Detailed history, physical examination and imaging, and high index of clinical suspicion are required to make an accurate diagnosis of this potentially life-threatening disease. There are several differential diagnoses of "S1-Q3-T3" signs on electrocardiogram (EKG) including but not limited to cor pulmonale, PE, and pneumothorax [3]. To our knowledge, there is no report in the medical literature in patients with atrial myxoma in the absence of PE or any other lung pathology presenting with this EKG pattern. We present a case of a 46-year-old female patient that was admitted for a syncope workup and was found to have left atrial myxoma (LAM). Initial EKG and laboratory results raised high suspicion for PE, but imaging tests only confirmed newly diagnosed LAM.

\section{Case Report}

\section{Investigations}

A 46-year-old female patient with no past medical history presented to the emergency department (ED) with worsening dizziness and weakness resulting in a presyncope. The episode was witnessed by her family members who reported that she reached down to tie her shoelaces and upon rising endorsed dizziness, isolated paresthesias in the right hand, and dysarthria for approximately $15 \mathrm{~min}$. Upon presentation to the ED, her symptoms had largely resolved. The patient was awake, alert, and oriented to person, place, and time. Physical examination revealed minor numbness in the thumb of the right hand. Otherwise, cranial nerves II-XII were grossly intact. There were no signs of pronator drift and the patient had intact muscle strength with flexion and extension of the upper and lower extremities. Cardiovascular examination was significant for a loud P2 with tumor plop at the cardiac apex as can be appreciated in Supplementary Material 1 (www.journalmc.org). Vital signs on admission were as follows: heart rate of 103 beats per minute, blood pressure of 173/105 $\mathrm{mm} \mathrm{Hg}$, temperature of $36.7^{\circ} \mathrm{C}$, respiratory rate of 16 breaths per minute, and pulse oximetry of $94 \%$ on room air. 
Table 1. Laboratory Workup

\begin{tabular}{lll}
\hline Laboratory test & Result value & Reference range \\
\hline White blood cells & 7.2 & $4.5-11.0 \times 10^{3} / \mu \mathrm{L}$ \\
Hemoglobin & 15.1 & $12.0-16.0 \mathrm{~g} / \mathrm{dL}$ \\
Hematocrit & 44 & $35.0-48.0 \%$ \\
Mean corpuscular volume & 87.5 & $80.0-100.0 \mathrm{fL}$ \\
Platelet count & 320 & $140-450 \times 10^{3} / \mu \mathrm{L}$ \\
Sodium & 140 & $136-145 \mathrm{mmol} / \mathrm{L}$ \\
Potassium & 4 & $3.5-5.2 \mathrm{mmol} / \mathrm{L}$ \\
Chloride & 106 & $96-110 \mathrm{mmol} / \mathrm{L}$ \\
Glucose & $123(\mathrm{H})$ & $70-99 \mathrm{mg} / \mathrm{dL}$ \\
Blood urea nitrogen & 6 & $5-25 \mathrm{mg} / \mathrm{dL}$ \\
Albumin & 4.1 & $3.5-5.0 \mathrm{~g} / \mathrm{dL}$ \\
Total bilirubin & 0.6 & $0.2-1.3 \mathrm{mg} / \mathrm{dL}$ \\
Calcium & 9.6 & $8.5-10.5 \mathrm{mg} / \mathrm{dL}$ \\
Creatinine & 0.57 & $0.44-1.00 \mathrm{mg} / \mathrm{dL}$ \\
Alkaline phosphatase & 110 & $38-126 \mathrm{U} / \mathrm{L}$ \\
Total protein & 6.7 & $6.0-8.0 \mathrm{~g} / \mathrm{dL}$ \\
Aspartate aminotransferase & 25 & $10-42 \mathrm{U} / \mathrm{L}$ \\
Anion gap ratio & 1.6 & $>1.0$ \\
Carbon dioxide & 26 & $24-31 \mathrm{mmol} / \mathrm{L}$ \\
Anion gap & 8 & $5-13 \mathrm{mmol} / \mathrm{L}$ \\
Alanine aminotransferase & 20 & $10-60 \mathrm{U} / \mathrm{L}$ \\
Glomerular filtration rate & $>60$ & $>60 \mathrm{~mL} / \mathrm{min} / 1.73 \mathrm{~m}{ }^{2}$ \\
Troponin I & $0.04(\mathrm{H})$ & $<0.04 \mathrm{ng} / \mathrm{mL}$ \\
\hline & & \\
\hline
\end{tabular}

\section{Diagnosis}

Laboratory data, as summarized in Table 1, including complete blood count, complete metabolic panel, and troponin were significant only for borderline elevation of troponin level of 0.04 $\mathrm{ng} / \mathrm{mL}$ (reference $<0.04 \mathrm{ng} / \mathrm{mL}$ ). EKG was significant for sinus tachycardia, left axis deviation with no signs of fascicular block, incomplete right bundle branch block with T-wave inversions in the inferior leads, and a classic "S1-T3-Q3"' pattern (Fig. 1). In the setting of mildly elevated troponins and characteristic EKG pattern, there was concern for PE. A reflex chest computed tomography angiogram (CTA) was negative for PE; however, it did reveal a $1.6 \times 3.6 \mathrm{~cm}$ filling defect in the left atrium (Fig. 2). We continued to trend the troponin and it had peaked to $0.16 \mathrm{ng} / \mathrm{mL}$ approximately $4 \mathrm{~h}$ later. Transthoracic echocardiogram (TTE) results revealed a $1.6 \times 3.4 \mathrm{~cm}$ mass fixed to the mitral leaflet (Fig. 3a). M-mode confirmed the localization of the mass to the mitral valve (Fig. 3b). Definity scan confirmed the mass but did not reveal any further masses within the left ventricle (Fig. 3c). The extent of outflow tract obstruction is demonstrated by Fig. 3d).

\section{Treatment}

Given the presenting symptoms and laboratory data, there was concern for a transient ischemic attack with underlying non-ST elevation myocardial infarction, thus the patient was administered $324 \mathrm{mg}$ of aspirin and high intensity atorvastatin. Maintenance intravenous fluids were ordered to promote volume expansion of the heart in the setting of a mobile mass and prevent left ventricular outflow tract obstruction. Systemic anticoagulation was initially deferred due to concern for thromboembolic masses to the brain. Although the initial CT scan of the head was negative, magnetic resonance imaging (MRI) of the brain revealed findings consistent with multiple bilateral microinfarcts in a vascular distribution. After further consultation with neurology and cardiothoracic surgery, it was deemed that the benefits of initiating anticoagulation with a heparin drip outweighed the risks of intracranial hemorrhaging given the small size of the infarcts. The patient underwent further evaluation including a left heart catheterization to rule out coronary artery disease in addition to cardiothoracic surgery evaluation for mass removal. Cardiac catheterization was negative for significant obstructive coronary artery disease.

\section{Follow-up and outcomes}

The patient ultimately underwent mass removal by cardiothoracic surgery and had an uncomplicated hospital course. The mass was sent for histopathological evaluation. Tissue evaluation was significant for a myxoid neoplasm that was composed of spindle shaped cells and capillaries on a myxoid stroma consistent with an atrial myxoma. The neurological symptoms had resolved, and the patient was discharged safely to home.

\section{Discussion}

Atrial myxomas were first reported as "simple vascular growth" in medical literature in 1845 followed by the first successful resection in $1955[4,5]$. Atrial myxomas occur with a prevalence of 1 per 10,000 people and often occur more frequently in women [2]. Atrial myxomas can be familial or sporadic in origin. They can occur at any age but are most often found in patients between the age of 20 and 59 years $[3,6]$. The clinical presentation varies greatly as patients can be asymptomatic or present with a myriad of findings including constitutional symptoms (fever, chills, and malaise), thromboembolic phenomena, or cardiogenic shock resulting in sudden cardiac death [6]. Given the potentially life-threatening complications, a detailed history, physical examination, imaging findings, and clinical suspicion are critical in early diagnosis and intervention. The severity of thromboembolic complications usually depends on the location of the tumor and size. Right-sided myxomas usually cause PE whereas left-sided myxomas can result in systemic embolic infarcts (stroke, splenic, or renal infarcts). Cardioembolic strokes account for approximately $30 \%$ of all ischemic strokes due to emboli, $0.5 \%$ of which are further attributed to atrial myxomas [7].

Cardiac myxomas are often pedunculated and can have 


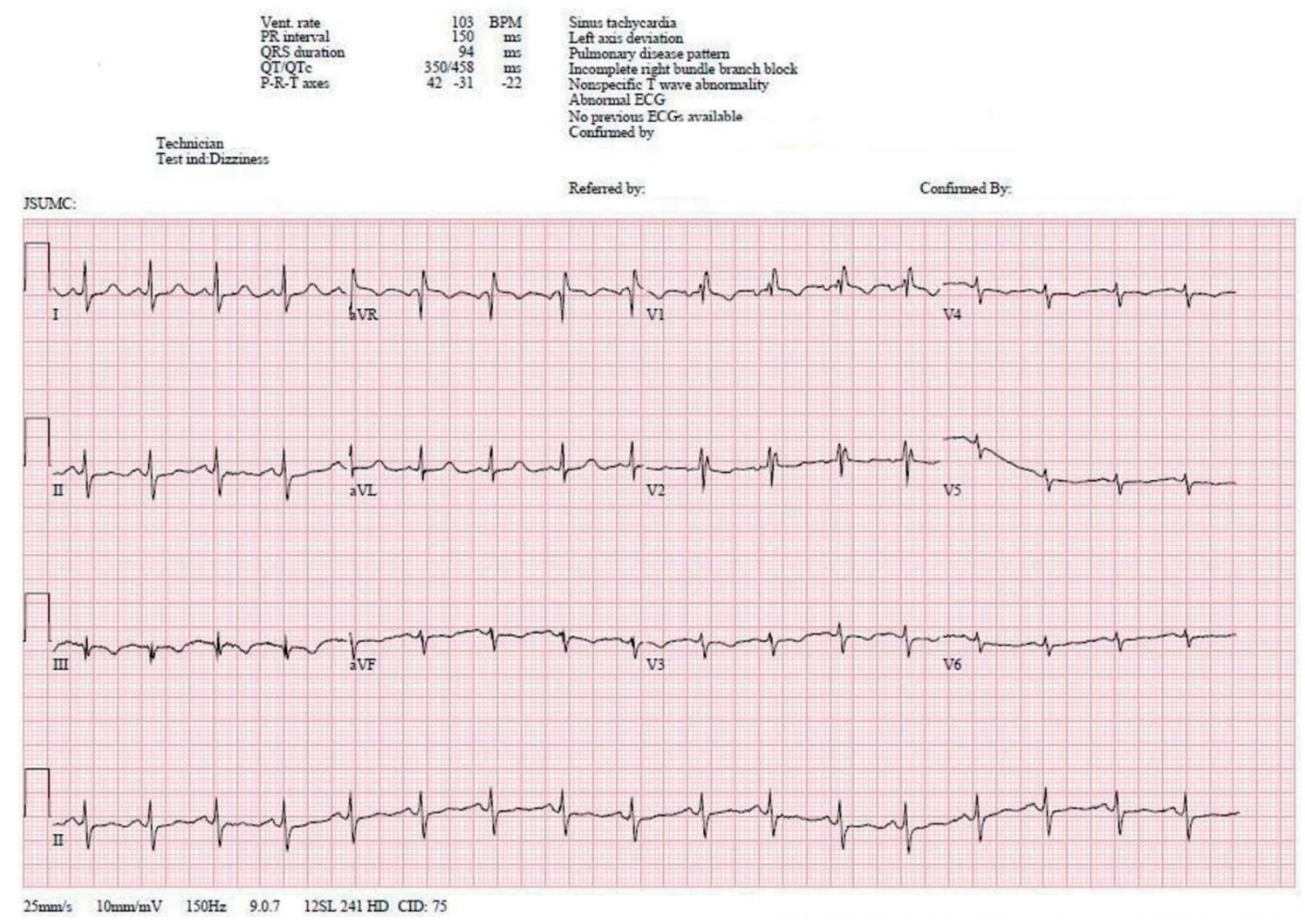

Figure 1. The 12-lead EKG obtained on initial evaluation is significant for sinus tachycardia with left axis deviation. An incomplete right bundle branch is present given by the RSR' pattern in leads V1-V2 in the setting of a QRS of 94 ms. The EKG is also significant for an S1-Q3-T3 pattern. EKG: electrocardiogram.

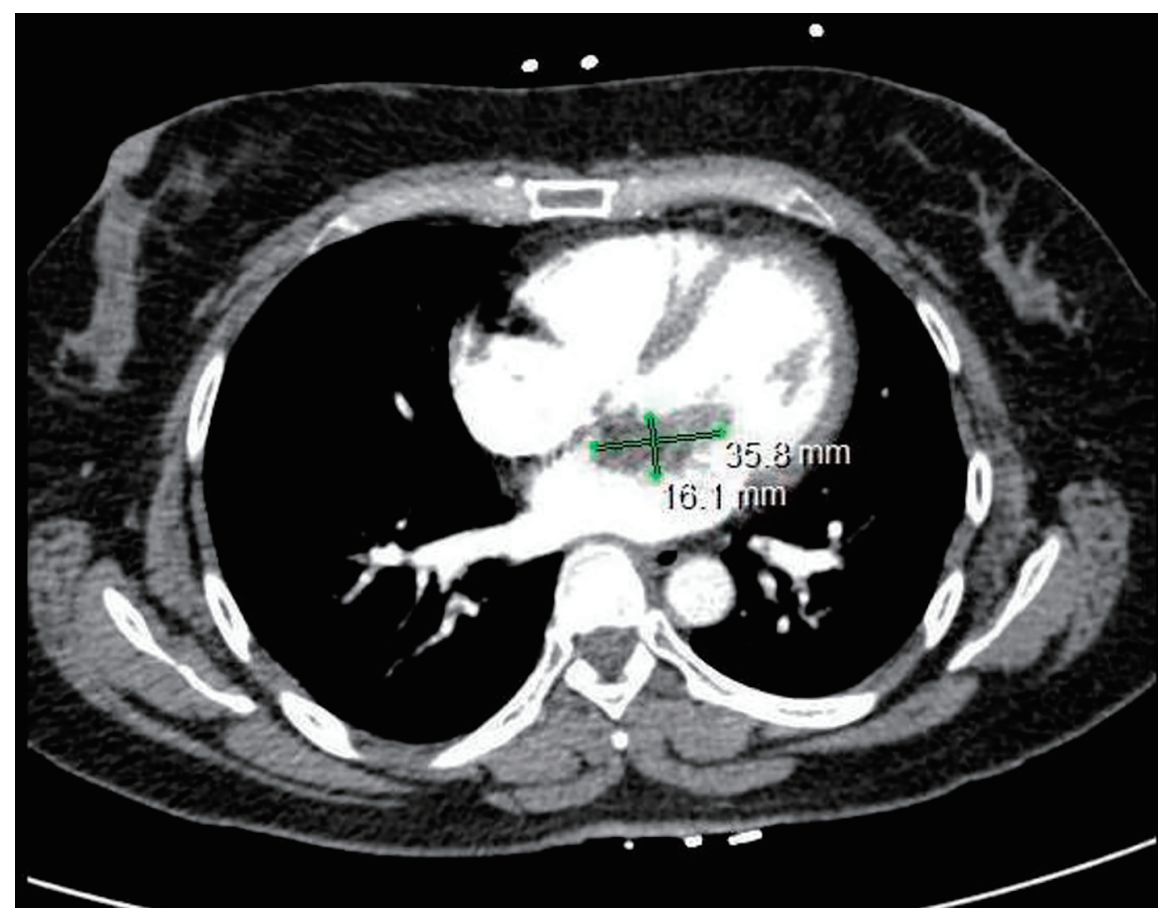

Figure 2. Computed tomography scan of the chest is significant for a filling defect within the left atrium that measures approximately $3.6 \times 1.6 \mathrm{~cm}$. 


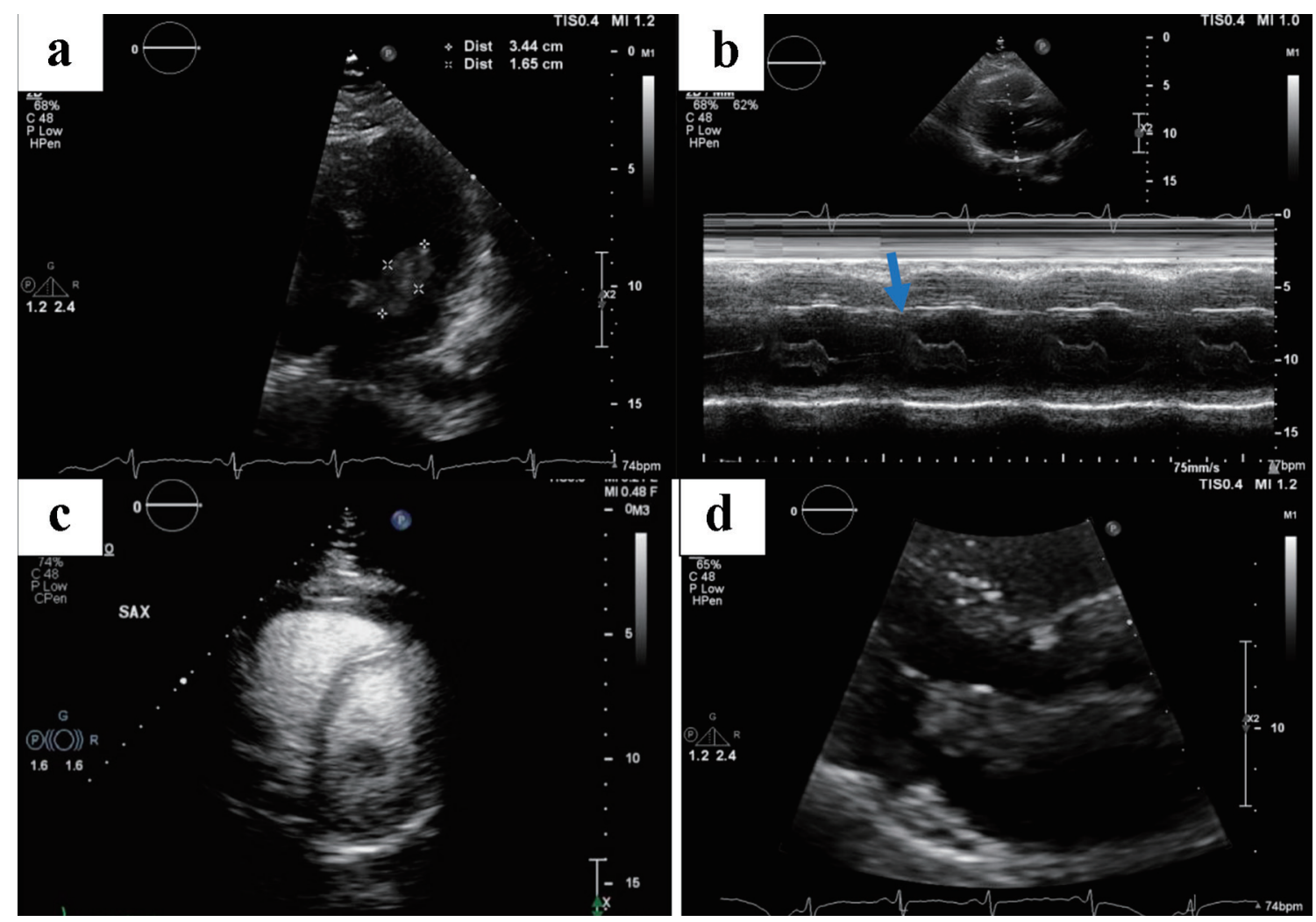

Figure 3. Serial images obtained by a transthoracic echocardiogram. (a) The size of the affixed mass measuring $1.6 \times 3.4 \mathrm{~cm}$. (b) The mass is affixed to the mitral valve (blue arrow) as seen on M-mode. (c) No other filling defects in the left ventricle aside from the previously described mass. (d) The extent of outflow tract obstruction.

a round, stellate, spindle-shaped, oval or polypoid morphology, with a smooth or lobulated surface [3]. Histologically, it is composed of polyclonal cells mixed in glycosaminoglycans $[3,6]$. The size and rate of growth is dependent on the vascular endothelial growth factor (VEGF) produced by polyclonal cells [8]. They can grow up to $4-5 \mathrm{~cm}$, with a larger size increasing the risk of symptoms due to its friability and obstructive effect. Clinically, as appreciated in our patient, a characteristic clinical exam finding is a mid-diastolic rumble murmur or "tumor plop" that is heard best at the cardiac apex during auscultation [6].

EKG can reveal clinical clues that can assist in diagnosis. Several reports have revealed non-specific EKG changes such as atrial fibrillation, atrial flutter, and left atrial hypertrophy in about $20-40 \%$ of patients with cardiac myxoma [9]. Left atrial hypertrophy is the most common EKG findings in these patients [9]. The classic S1-Q3-T3 sign on an EKG strip is often associated with PE, but can also be found in other pulmonary diseases such as pneumothorax, bronchospasm, and cor pulmonale [6]. EKG evaluation is a cornerstone in the evaluation of patients that present with syncope. At the time of our writing, there are no reported cases of patients diagnosed with atrial myxoma without PE that have an S1-Q3-T3 pattern on EKG.

Initially, atrial myxomas were only diagnosed on autopsy. However, with the advent of echocardiography and more advanced imaging modalities, they have become much easier to diagnose. They can be often found incidentally on CT and MRI scans. Although TTE is often adequate for diagnosis, a transesophageal echocardiogram is preferred as it has a better sensitivity and can better characterize smaller tumors [10]. Unlike MRI and CT imaging, an echocardiogram can also provide critical information on hemodynamic complications related to the tumor mass. Once diagnosed, surgical removal is the mainstay of treatment. The rate of recurrence is reported to be $2-3 \%$ unless that patient is diagnosed with a familial disorder at which point recurrence is much greater [11]. Carney complex, one such familial disorder, is characterized by cardiac and mucocutaneous myxomas in addition to hyperpigmentation and schwannomas. In these patients, there is still a risk of recurrence even after surgical resection [12]. Outcomes after surgery intrinsically depend on the extent of disease and acumen of the surgeon; however, post-operative complications including mitral valve insufficiency and left atrial dissection have been reported $[12,13]$.

\section{Learning points}

PCTs are rare but when diagnosed they are usually due to cardiac myxomas. Cardiac myxomas are often diagnosed in patients between the age of 20 and 59 years. Cardiac myxomas are often dubbed "the great masquerader" in medical literature as patients can present with a wide range of other- 
wise non-specific symptoms. Patients can be asymptomatic or present with sequela of thromboembolic events. Our patient presented with a syncopal episode and was found to have an "S1-Q3-T3" pattern on EKG, a finding that is often associated with PE. Although described as a benign tumor, the morbidity and mortality of cardiac myxomas stems from the risk of thromboembolism and hemodynamic collapse secondary to left ventricular outflow obstruction. Echocardiography, preferably transesophageal, remains the gold standard as it is better at characterizing the morphology of the tumor. Surgery is the mainstay of treatment and recurrence remains low if the patient does not have an underlying predisposition.

\section{Supplementary Material}

Suppl 1. A transthoracic stethoscope with audio recording capability was used to record the heart sounds. Partial obstruction of the mitral valve's orifice due to myxoma leads to mitral stenosis. Mitral stenosis causes loud S1 and diastolic low-frequency murmur. The phonogram illustrates an attenuated S1. The first sound that we hear will be labeled as the first beat. We can occasionally appreciate a low frequency, diastolic murmur before the seventh and 11 th beat.

\section{Acknowledgments}

None to declare.

\section{Financial Disclosure}

None to declare.

\section{Conflict of Interest}

None to declare.

\section{Informed Consent}

Informed consent was obtained.

\section{Author Contributions}

Anton Mararenko: preparation of manuscript. Ndausung Udongwo: preparation of manuscript. Dhaval Desai: preparation of manuscript. Matthew Schoenfeld: senior author, operating attending.

\section{Data Availability}

The data supporting the finding of this study are available from the corresponding author upon reasonable request.

\section{References}

1. Pandey AC, Carey JJ, Thompson JL. Right atrial myxoma presenting as a pulmonary embolism in a 32-year-old female. JRSM Cardiovasc Dis. 2019;8:2048004018817606.

2. Poterucha TJ, Kochav J, O'Connor DS, Rosner GF. Cardiac tumors: clinical presentation, diagnosis, and management. Curr Treat Options Oncol. 2019;20(8):66.

3. Arshad H, Khan RR, Khaja M. Case report of S1Q3T3 electrocardiographic abnormality in a pregnant asthmatic patient during acute bronchospasm. Am J Case Rep. 2017;18:110-113.

4. King, et al. On simple vacular growths in the left auricle of the heart. The Lancet. 1845;46(1155):428-429.

5. Glover RP. Late results of mitral commissurotomy. In: Lam CR, ed. Henry Ford Hospital international symposium on cardiovascular surgery: studies in physiology, diagnosis and techniques: proceedings of the symposium, Henry Ford Hospital, Detroit (Michigan), March 1955. Philadelphia: WB Saunders; 1955. p. 202e11.

6. Obagi A, Desai D, Mazahir U, Johnson D, Berger L. Large right atrial myxoma presenting as bilateral pulmonary embolism. Cureus. 2021;13(6):e15889.

7. Kalra DK, Hemu M, Kyung S, Reddy V, Rao A, Volgman A. Atrial myxoma-the Great Masquerader. QJM. 2019;112(5):363-364.

8. Maleszewski JJ, Anavekar NS, Moynihan TJ, Klarich KW. Pathology, imaging, and treatment of cardiac tumours. Nat Rev Cardiol. 2017;14(9):536-549.

9. Nantha YS, Malek SA. Silent left large atrial myxoma: a patient with serial electrocardiogram variation. Korean J Fam Med. 2017;38(4):229-232.

10. Bernatchez J, Gaudreault V, Vincent G, Rheaume P. Left atrial myxoma presenting as an embolic shower: a case report and review of literature. Ann Vasc Surg. 2018;53:266. e220.

11. Mendoza C, Bernstein E, Ferreira A. Multiple recurrences of nonfamilial cardiac myxomas: a report of two cases. Tex Heart Inst J. 2007;34(2):236-239.

12. Orozco Vinasco DM, Abello Sanchez M, Osorio Esquivel JE. [Severe mitral regurgitation following resection of a giant atrial myxoma: Case report and literature review]. Rev Esp Anestesiol Reanim. 2013;60(7):403-406.

13. Niyogi SG, Kumar B, Singh H, Biswas I. Left atrial dissection and rupture following excision of left atrial myxoma: role of transesophageal echocardiography. J Cardiothorac Vasc Anesth. 2020;34(10):2823-2826. 